\title{
Diagnostic utility of computed tomographic angiography in dogs with portal vein thrombosis
}

\author{
Keita SATO ${ }^{1) \#, ~ Y u m i ~ S A K A M O T O ~}{ }^{1) \#, ~ M a n a b u ~ S A K A I ~}{ }^{1) *}$, Chieko ISHIKAWA ${ }^{1 \text {, }}$ \\ Megu NAKAZAWA ${ }^{1)}$, Chieh-Jen CHENG $^{1)}$, Toshihiro WATARI ${ }^{1)}$ and \\ Tomohiro NAKAYAMA ${ }^{1)}$
}

${ }^{1)}$ Department of Veterinary Medicine, College of Bioresource Sciences, Nihon University, 1866 Kameino, Fujisawa, Kanagawa 252-0880, Japan

J. Vet. Med. Sci.

82(10): 1421-1427, 2020

doi: 10.1292/jvms.20-0226

Received: 20 April 2020

Accepted: 5 August 2020

Advanced Epub:

18 August 2020

\begin{abstract}
Computed tomographic (CT) angiography, the gold standard for diagnosing portal vein thrombosis (PVT) in humans, is poorly documented in dogs. Therefore, we retrospectively reviewed dogs with PVT diagnosed by CT angiography. Medical records of 13 client-owned dogs diagnosed with PVT by CT angiography were reviewed. All dogs had chronic PVT, and the most frequent clinical sign was vomiting $(5 / 13)$, with pancreatitis the most frequent concurrent disease (6/13). All dogs tested for plasma D-dimer concentration (12/12) revealed elevated levels. On CT angiography, a thrombus was detected as a non-contrast enhancement structure in the portal vessel of 13 dogs. There was no evidence of complete obstruction of the portal vein in any of the dogs. The median luminal filling of the portal vein was $60.4 \%$. The thrombus extension was variable among dogs, with a median of $34.9 \mathrm{~mm}$. CT angiography identified the thrombus in the main portal vein of $12 / 13$ dogs and multiple thrombus formation other than the main portal vein in $9 / 13$ dogs. CT angiography provided specific information such as detecting the presence, location, and number of PVT in dogs. Therefore, CT angiography might be useful for the diagnosis and follow-up evaluation of PVT in dogs.

KEY WORDS: canine, computed tomography, d-dimer, portal vein thrombosis
\end{abstract}

Portal vein thrombosis (PVT) is a rare condition in dogs $[6,11,18,22]$. However, recent studies suggest that its incidence and prevalence in conditions such as pancreatitis might be highly underestimated [7]. Portal vein thrombosis refers to partial or total obstruction of the portal blood flow in the portal venous system [17, 18]. In dogs, obstruction of the portal vein may lead to presinusoidal portal hypertension, ascites and acquired portosystemic collaterals (APSCs) [1, 2, 22]. Conditions such as administration of glucocorticoids, hepatic diseases, systemic inflammatory response syndrome (SIRS), previous invasive procedures involving the portal vein, protein-losing diseases, and pancreatitis were associated with PVT [6, 7, 11, 18, 22].

Canine PVT is classified into acute or chronic, based on clinical presentation: PVT with acute abdominal pain, hypovolemic shock, or both is classified as acute, while the remaining cases were classified as chronic [18]. Dogs with chronic PVT are more likely to survive longer than those with acute PVT [18]. Additionally, dogs without PVT have a shorter hospitalization period in comparison with dogs with PVT [7]. However, long-term follow-up data concerning the course of PVT is limited in dogs.

Computed tomographic (CT) angiography is the gold standard for definitive diagnosis of PVT in humans because of its reliability to determine thrombus extent and intestinal infarction [5, 17]. Additionally, it is used for diagnosis and preoperative surgical planning for carvernous transformation of the portal vein, which is a common feature in chronic PVT [20, 26]. Furthermore, follow-up CT is performed to evaluate the course of the thrombus $[3,4,8]$. However, diagnosis in the majority of previous reports on dogs with PVT has been performed by abdominal ultrasound, despite the fact that this modality is considered operator-dependent $[6,11,18,22]$. Recently, CT was reported to be superior to abdominal ultrasound in detecting PVT in dogs with acute pancreatitis [7]. This report suggested the utility of CT; however, information concerning follow-up or concurrent diseases other than pancreatitis related to PVT was limited.

The aim of the present study was to investigate the application of CT imaging to characterize canine PVT and the utility of CT angiography in the diagnosis of PVT in dogs. 


\section{MATERIALS AND METHODS}

\section{Dogs}

The medical record database of Nihon University Animal Medical Center (ANMEC) was reviewed retrospectively to identify cases with the following inclusion criteria: dogs diagnosed with PVT on CT angiography, from 2008 to 2018, and presenting complete medical records. The following medical information was obtained from each record: breed, sex, age, concurrent conditions, clinical signs, clinicopathological data, diagnostic imaging findings, administration of glucocorticoids prior to diagnosis, treatment, and outcome. Based on previously reported criteria, pre-existing diseases were recorded, including pancreatitis, proteinlosing enteropathy (PLE), disseminated intravascular coagulation (DIC), SIRS, and hepatopathy [11, 12]. In addition, the cases' history of prior abdominal surgery involving the portal system was recorded. Follow-up imaging was performed at our facility.

\section{Abdominal ultrasound and CT angiography}

Abdominal ultrasonography was performed by one examiner (M.S) using a B-mode ultrasonographic scanner (ProSound $\alpha 7$ or EUB-6500, Hitachi, Ltd., Tokyo, Japan), with a microconvex transducer (4-8 MHz). The majority of the images were obtained by the right intercostal approach, and the location of the thrombus, number, and echogenicity was evaluated.

CT angiography was performed under general anesthesia. All dogs were maintained under controlled ventilation, and the CT scanning was performed with breath-holding. The dogs were positioned in ventral recumbency and imaged using a 16- or 320-slice multi-detector CT scanner (Aquilion 16 or Aquilion ONE, Canon Medical Systems, Otawara, Japan). Iohexol (Ioverin 300, Teva Pharma Japan Inc., Nagoya, Japan) was used as the contrast medium, which was administered at a dose of $2.5 \mathrm{ml} / \mathrm{kg}(750 \mathrm{mgI} /$ $\mathrm{kg}$ ) via the cephalic vein with a power injector. The injection time was fixed at $15 \mathrm{sec}$ with a maximum injection speed of $3 \mathrm{~m} l /$ sec. Contrast enhancement of the aorta was monitored until the enhancement reached 150 Hounsfield units (HU) using a bolustracking program (Real Prep., Canon Medical Systems). CT scans were performed immediately after, $20 \mathrm{sec}$, and $120 \mathrm{sec}$ after aortic enhancement of $150 \mathrm{HU}$. These scanning points represented the arterial phase, portal venous phase, and delayed phases, respectively.

The scan parameters for the 16-slice CT were as follows: rotation time, $0.5 \mathrm{sec}$; slice thickness, $1-2 \mathrm{~mm}$; reconstruction interval, 0.5-1 mm; table speed, 16-32 mm/rotation; helical pitch, 16.0; X-ray tube potential, $120 \mathrm{kV}$; and X-ray tube current, $150 \mathrm{~mA}$. The scan parameters for the 320 -slice CT were as follows: rotation time, $0.5 \mathrm{sec}$; slice thickness, $0.5 \mathrm{~mm}$; reconstruction interval, $0.5 \mathrm{~mm}$; helical pitch, 65.0 ; X-ray tube potential, $120 \mathrm{kV}$; and X-ray tube current, max $350 \mathrm{~mA}$, respectively.

\section{Image analysis}

The obtained CT images were retrospectively reviewed by two observers using a workstation (Virtual Place, Canon Medical Systems). The morphological structure of the portal venous system was evaluated by maximum intensity projection, volume rendering images, curved planar reformation, and 2D multiplanar images. The location of the thrombus was distinguished as previously reported $[18,22]$; the main portal vein, right and left branches of the intrahepatic portal vein, gastroduodenal vein, splenic vein, and the mesenteric vein were identified. The following features were measured: extent measurement of the thrombus, presence of cavernous transformation of the portal vein, and the luminal filling of the portal vein by the thrombus. On the curved planar reformation (Fig. 1), the extent measurement and luminal filling were calculated as previously reported [19]. Additionally, APSCs were detected by volume rendering images. The presence of cavernous transformation was analyzed by maximum intensity projection. Thoracic CT images were analyzed in each dog to confirm remote thrombus formation in the thoracic vasculature.

\section{Statistical analysis}

Data were expressed as median (range). Differences in plasma D-dimer concentration between dogs with a single PVT and dogs with thrombi at multiple locations were statistically analysed using the Mann Whitney test. The data was analyzed using GraphPad PRISM for Mac OS X version 5.0b (GraphPad Software Inc., La Jolla, CA, USA). Differences were considered significant at $P<0.05$.

\section{RESULTS}

Our review of the medical records database revealed 13 dogs with PVT diagnosed by CT angiography (Table 1). Since none of the dogs presented with abdominal pain or hypovolemic shock, all cases were classified as chronic PVT. Breeds of the 13 dogs included Shiba (2/13), mixed breed (2/13), Cavalier King Charles Spaniel (1/13), Kooikerhondje (1/13), Labrador Retriever (1/13), Miniature Dachshund (1/13), Miniature Schnauzer (1/13), Pug (1/13), Standard Poodle (1/13), Toy Poodle (1/13), and Yorkshire Terrier (1/13). The median body weight and age were $9.5 \mathrm{~kg}$ (range, 2.7-28.1 kg) and 11.0 years (range, $4.3-14.2$ years), respectively. There were six spayed females, four neutered males, and three intact males. Five dogs were symptomatic at their first presentation at our hospital and presented with vomiting (5/5), anorexia (4/5), lethargy (3/5), diarrhea (1/5), and seizure (1/5). Eight dogs were asymptomatic. Pre-existing diseases and conditions suspected to be associated with PVT were identified in all dogs. More than one concurrent condition was identified in nine dogs. Six dogs had pancreatitis. Other conditions were PLE (4/13), SIRS (3/13), chronic hepatitis (2/13), DIC (1/13), and previous splenectomy (1/13). Eight dogs were receiving glucocorticoid therapy for a median of 20 days (range, 7-599 days). The dosage ranged from 0.04 to $0.07 \mathrm{mg} / \mathrm{kg} / \mathrm{day}$ for dexamethasone (1/8) and 0.5 to $2.0 \mathrm{mg} / \mathrm{kg}$ /day for prednisolone (7/8), respectively. Of the $13 \mathrm{dogs}$, two dogs were diagnosed with PVT during their hospitalization 

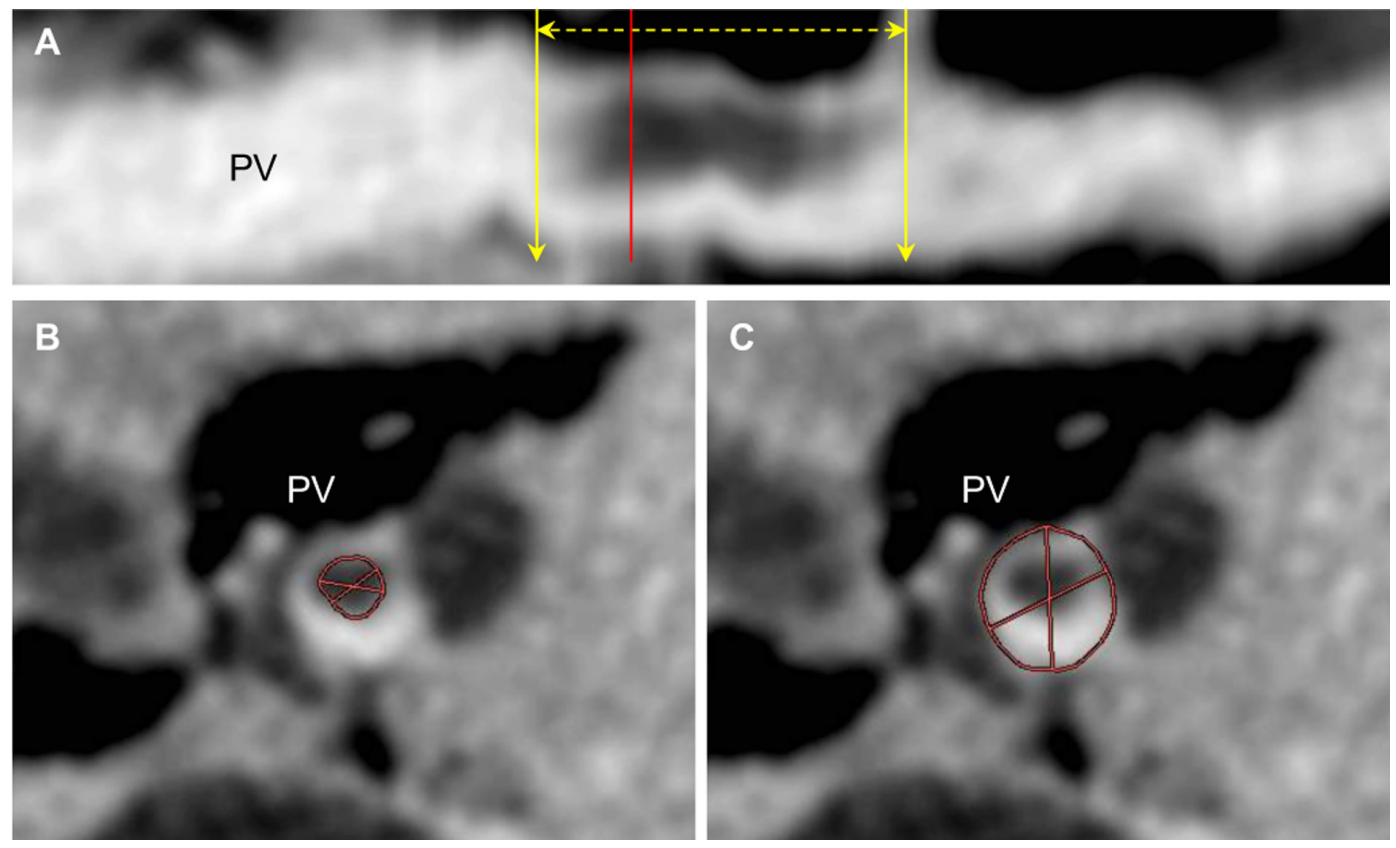

Fig. 1. Curved planar reformation (CPR) images of canine portal vein thrombosis (Dog 7) obtained via CT angiography. (A) The yellow dashed line indicates the length of the thrombus between the cranial (left), and caudal (right) end of the thrombus (two yellow arrows). The red line represents the point of the maximum diameter of the thrombus (B, C). The maximum areas of the thrombus and the portal vein were calculated using orthogonal cross-sectional CPR images of the same dog. The luminal filling of the thrombus was measured by dividing the maximum area of the thrombus (B) by the portal vein area (C). PV, portal vein.

Table 1. Summary of signalment, clinical signs, pre-existing conditions, and D-dimer concentration of 13 dogs with portal vein thrombosis

\begin{tabular}{|c|c|c|c|c|c|c|c|}
\hline Dog & Breed & $\begin{array}{l}\text { Age } \\
\text { (Years) }\end{array}$ & $\begin{array}{c}\text { Body } \\
\text { weight }(\mathrm{kg})\end{array}$ & Sex & Clinical signs & Pre-existing conditions & $\begin{array}{l}\text { D-dimer } \\
(\mu \mathrm{g} / \mathrm{m} l)^{\mathrm{a})}\end{array}$ \\
\hline 1 & Miniature Schnauzer & 11.0 & 8.8 & $\mathrm{MC}$ & Vomiting, anorexia, lethargy & Pancreatitis & 7.6 \\
\hline 2 & Cavalier King Charles Spaniel & 12.1 & 9.7 & $\mathrm{MC}$ & Asymptomatic & Pancreatitis & 3.9 \\
\hline 3 & Yorkshire Terrier & 4.3 & 2.7 & M & Asymptomatic & Pancreatitis, SIRS & 28.9 \\
\hline 4 & Toy Poodle & 8.4 & 4.3 & M & Vomiting, anorexia & Pancreatitis, SIRS & 4.0 \\
\hline 5 & $\operatorname{Mix}$ & 14.2 & 13.4 & M & Asymptomatic & Pancreatitis, glucocorticoid therapy & 3.6 \\
\hline 6 & Shiba & 9.0 & 13.2 & FS & Vomiting, anorexia, diarrhea & $\begin{array}{l}\text { Pancreatitis, SIRS, PLE, } \\
\text { glucocorticoid therapy }\end{array}$ & NT \\
\hline 7 & Kooikerhondje & 11.2 & 9.5 & $\mathrm{MC}$ & Vomiting, lethargy & PLE, glucocorticoid therapy & 1.1 \\
\hline 8 & Pug & 8.9 & 7.0 & FS & Asymptomatic & PLE, glucocorticoid therapy & 6.4 \\
\hline 9 & Miniature Dachshund & 10.9 & 3.7 & $\mathrm{MC}$ & Asymptomatic & PLE, glucocorticoid therapy & 30.1 \\
\hline 10 & Mix & 11.5 & 25.8 & FS & Asymptomatic & $\begin{array}{l}\text { Chronic hepatitis, glucocorticoid } \\
\text { therapy }\end{array}$ & 2.9 \\
\hline 11 & Labrador Retriever & 7.5 & 28.1 & FS & Asymptomatic & $\begin{array}{l}\text { Chronic hepatitis, glucocorticoid } \\
\text { therapy, DIC }\end{array}$ & 10.3 \\
\hline 12 & Standard Poodle & 13.5 & 21.2 & FS & Asymptomatic & Previous splenectomy & 11.3 \\
\hline 13 & Shiba & 12.0 & 9.0 & FS & $\begin{array}{l}\text { Vomiting, anorexia, lethargy, } \\
\text { seizure }\end{array}$ & Glucocorticoid therapy & 5.0 \\
\hline
\end{tabular}

MC, male-castrated; M, male; FS, female-spayed; SIRS, systemic inflammatory response syndrome; PLE, protein-losing enteropathy; DIC, disseminated intravascular coagulation; NT, not tested. a) Reference range; $<1.0 \mu \mathrm{g} / \mathrm{ml}$.

due to pancreatitis, and the other dogs were diagnosed at the initial referral to our hospital. D-dimer concentrations were measured in 12 dogs and were elevated in all dogs. Additionally, it was statistically higher in dogs with multiple thrombi (median, $9.0 \mu \mathrm{g} / \mathrm{m} l$ ) compared to dogs with a single thrombus in the portal vein (median; $3.7 \mu \mathrm{g} / \mathrm{ml}, P=0.0283$ ).

The summary of abdominal ultrasound and CT features is shown in Table 2. PVT was found in eight dogs on the initial ultrasound examination. Thrombus echogenicity were homogeneous in five dogs and hyperechoic in three dogs. The thrombus was located at the level of the porta hepatis in eight dogs. Splenic vein thrombosis was concurrently detected in one dog. Ascites and APSCs were confirmed in six and three dogs, respectively. In the five dogs in which PVT was not detected by ultrasound, one dog had severe ascites. Moreover, two dogs were large-breed dogs. These five dogs without PVT in abdominal ultrasound underwent 
Table 2. Summary of abdominal ultrasound and computed tomography features of 13 dogs with portal vein thrombosis

\begin{tabular}{lcc}
\hline \multicolumn{1}{c}{ Variable } & $\begin{array}{c}\text { Abdominal ultrasound } \\
\mathrm{n}(\%)\end{array}$ & $\begin{array}{c}\text { Computed tomography } \\
\mathrm{n}(\%)\end{array}$ \\
\hline Portal vein thrombosis & $8(62)$ & $13(100)$ \\
Main portal vein & $8(62)$ & $12(92)$ \\
Right branch of intrahepatic portal vein & $0(0)$ & $11(85)$ \\
Left branch of intrahepatic portal vein & $0(0)$ & $9(69)$ \\
Gastroduodenal vein thrombosis & $0(0)$ & $4(31)$ \\
Splenic vein thrombosis & $1(8)$ & $5(38)$ \\
Mesenteric vein thrombosis & $0(0)$ & $2(15)$ \\
\hline Thoracic thrombus & $\mathrm{NA}$ & $3(23)$ \\
Pulmonary thromboembolism & & $3(23)$ \\
Pulmonary vein thrombosis & & $1(8)$ \\
Jugular vein thrombosis & $1(8)$ & $1(8)$ \\
\hline Thrombosis of multiple locations & $3(23)$ & $9(69)$ \\
Acquired portosystemic collaterals & $1(8)$ & $6(46)$ \\
Asymmetry of liver lobes & & $2(15)$ \\
\hline
\end{tabular}

NA, not applicable.

CT examination for further evaluation of suspected thrombus formation from high D-dimer concentration (4/5), splenic mass (1/5), and suspected portosystemic shunts (1/5). In the one dog without high D-dimer concentration, D-dimer was not measured.

CT angiography was performed without complications in all dogs. Each thrombus was detected as a non-contrast enhancement structure in the portal vein (Fig. 2A). The contrast enhancement between the thrombus and the wall of the blood vessel was clearly observed in the portal and in the delayed phases in all dogs. From these results, there was no evidence of complete obstruction of the portal vein in any of the dogs. The median luminal filling of the portal vein was $60.4 \%$ (range, 32.5-74.3\%). The thrombus extension varied among dogs, with a median of $34.9 \mathrm{~mm}$ (range, 11.8-207.2 mm). The thrombus of the main portal vein was the most frequently observed, with 12 dogs showing a thrombus in this vein. The one dog without a thrombus in the main portal vein had a small thrombus affecting the right and left branches of the intrahepatic portal vein. In total, multiple thrombus formation at sites other than the main portal vein was detected in nine dogs. Concurrent with the portal vein, the thrombus was detected in the splenic vein (5/13), gastroduodenal vein (4/13), and the mesenteric vein $(2 / 13)$. On thoracic evaluation, three dogs were detected to have pulmonary thromboembolism (PTE), and among the three dogs, additional thrombus formation in the left jugular vein and pulmonary vein was detected in one dog each. Liver lobe asymmetry and APSCs were detected in two and nine dogs, respectively (Fig. 2B and 2C). Cavernous transformation of the portal vein was observed in 11 dogs (Fig. 2D and 2E).

Of the eight dogs that had been receiving glucocorticoids prior to diagnosis of PVT, six continued to receive the drugs for PLE (4/6) and chronic hepatitis (2/6). Ten of the 13 dogs with PVT were treated with anticoagulant or antiplatelet therapy after the diagnosis of PVT. Nine dogs were treated with low molecular weight heparin (LMWH; 50-100 IU/kg subcutaneously, q12-24 hr; Fragmin, Pfizer Japan Inc., Tokyo, Japan), and one dog each with unfractionated heparin (100 IU/kg subcutaneously, q12 hr; Novo-Heparin, Mochida Pharmaceutical Co., Ltd., Tokyo, Japan) and clopidogrel (3.0 mg/kg orally, q24 hr; Clopidogrel, Sawai Pharmaceutical Co., Ltd., Osaka, Japan). Two dogs were prescribed ozagrel hydrochloride (5-10 mg/kg orally, q12-24 hr; Domenan, Kissei Pharmaceutical Co., Ltd., Tokyo, Japan) as antiplatelet therapy.

Follow-up data were obtained from all dogs with a median survival of 564 days (range, 6-1,504 days). Three dogs were alive at the censored time point. Four dogs died within 30 days after CT angiography. These dogs were detected multiple thrombus formation. The surviving nine dogs were reexamined at least once by abdominal ultrasound or CT angiography for follow-up evaluation of PVT. The follow-up ultrasound results showed complete resolution in one dog, partial resolution in two dogs. However, the PVT was unchanged in five dogs. In one dog, abdominal ultrasound failed to confirm the PVT in follow-up, thus this dog received follow-up examination by CT angiography. Among the five dogs in whom PVT was not detected on the initial ultrasound and alive for a follow-up examination, two were detected to have PVT in the follow-up ultrasound. Follow-up CT angiography was performed on two dogs with concurrent disease unidentified (Dog 13) and pancreatitis (Dog 2), respectively. Dog 13 was administered $\mathrm{LMWH}$, and presented with a marked decrease in the extension of the thrombus (62.4 $\mathrm{mm}$ to $13.8 \mathrm{~mm})$ and luminal filling $(69.4 \%$ to $39.9 \%)$ on day 63 . However, a mild increase in the thrombus size was detected as an extension of the thrombus (14.2 mm) and luminal filling (46.3\%) on day 183 (Fig. 3A-C). Additionally, the changes in luminal filling (61.0\% to $28.5 \%$ ) and increased organized area of the thrombi were detected in Dog 2 on day 448 (Fig. 3D-E).

\section{DISCUSSION}

CT angiography is a tool for definitive diagnosis of PVT in human medicine [5]. It is frequently used for the assessment of the portal venous branches that may not be easily assessed by ultrasound [5]. CT angiography has also been proven to be superior 

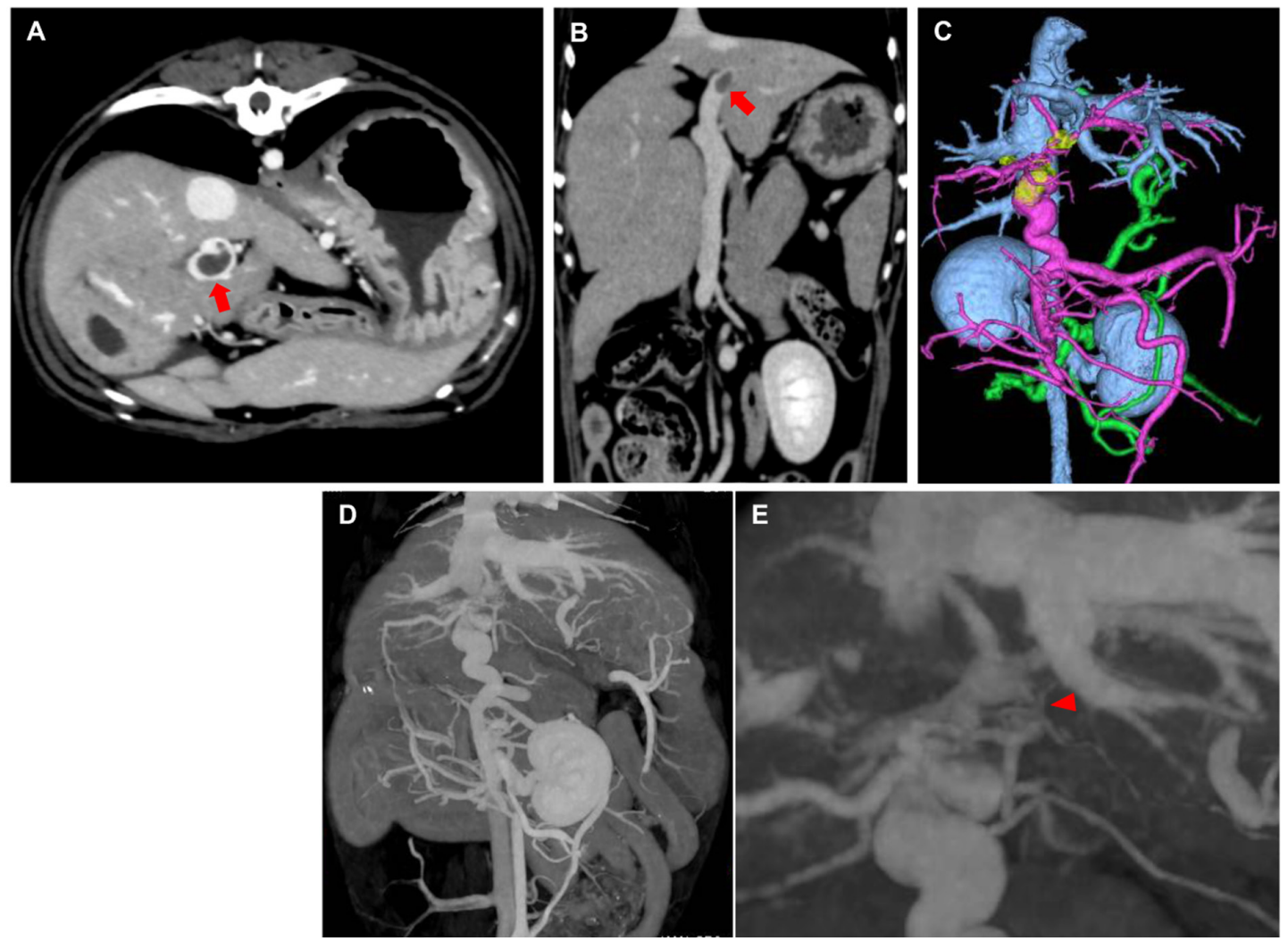

Fig. 2. CT angiography images of a dog with portal vein thrombosis (PVT). (A; Dog 8) Transverse image showing the PVT (arrow) within the main portal vein. The thrombosis was characterized as a hypoattenuating structure within the portal vein without contrast enhancement. (B; Dog 7) The dorsal aspect of CT images of a dog with a thrombus within the left branch of the intrahepatic portal vein (arrow). The asymmetry of the liver lobes was detectable with an atrophy of the left liver lobes. (C; Dog 8) Three-dimensional volume-rendered image of a dog with PVT. The venous system and kidneys appeared in blue, the portal system in purple, the acquired porrosystemic collaterals in light green, and the thrombosis in yellow. (D, E; Dog 8) Dorsal maximum intensity projection image of a dog with a cavernous transformation of the portal vein. The tortuous main portal vein due to the occlusion (D), and the small vessels bypassing the occlusion site (cavernous transformation, arrowhead) was detected (E).

to Doppler ultrasound for evaluation of thrombotic extension within the portal venous system of humans [5, 23]. Since contrastenhanced CT images enable visualization of the anatomical characteristics of the liver and the portal venous system in dogs, they have been used for diagnosis of hepatic diseases such as congenital portosystemic shunts and hepatic tumors [10, 16]. However, most dogs with PVT have been definitively diagnosed by autopsy, abdominal ultrasound, and surgery $[6,11,18,22]$. In veterinary medicine, there has been growing interest in the application of CT as an imaging modality to thoroughly describe the structure of the portal vein. The imaging features of cavernous transformation of the portal vein were recently reported using CT in seven dogs with PVT [20]. Additionally, the imaging features of acute pancreatitis were compared between abdominal ultrasound and CT, and a higher detectability of PVT using CT was found [7]. However, neither of the aforementioned studies described the morphological structure of the thrombus nor did they use CT as a follow-up tool. Therefore, at present, there is limited information considering CT angiography as a diagnostic method for PVT in dogs. Thus, in this study, we investigated the features of CT imaging of PVT in dogs.

In this study, CT angiography in dogs with PVT yielded high-quality images, providing specific features of PVT. The contrast enhancement between a thrombus and the wall of the blood vessel was clearly observed in the portal and the delayed phases, making the thrombus clearly distinguishable. The presence of an occlusion could be detected by evaluating contrast enhancement between the thrombus and the wall of the portal vein. Additionally, a cavernous transformation of the portal vein was detected in 11 dogs. Cavernous transformation of the portal vein refers to the angiographic appearance of the portal vein when numerous collateral vessels develop around the portal vein to overcome a complete or partial obstruction, and is considered a clinical feature of chronic PVT [5, 20, 24]. CT angiography is considered helpful for preoperative surgical planning in humans and for visualizing and characterizing these vessels in small animals $[20,26]$. Furthermore, CT angiography enabled visualization of the presence of thrombi in the portal venous system beyond the main portal branch. Notably, thrombus in the thoracic vasculature was diagnosed in our three dogs. Pulmonary thromboembolism is the obstruction of pulmonary vessels by a thrombus with an association with numerous thromboembolic conditions such as corticosteroid administration, surgery, PLE, protein-losing nephropathy, and pancreatitis [9]. Considering the fact that these underlying conditions in PTE are similar to those in PVT in dogs, the incidence of concurrent PTE may be highly under-recognized in past reports of PVT in dogs. Additionally, CT enabled visualization of the morphological changes in the hepatoportal system, including APSCs and liver lobe asymmetry. Portosystemic collateral formation 

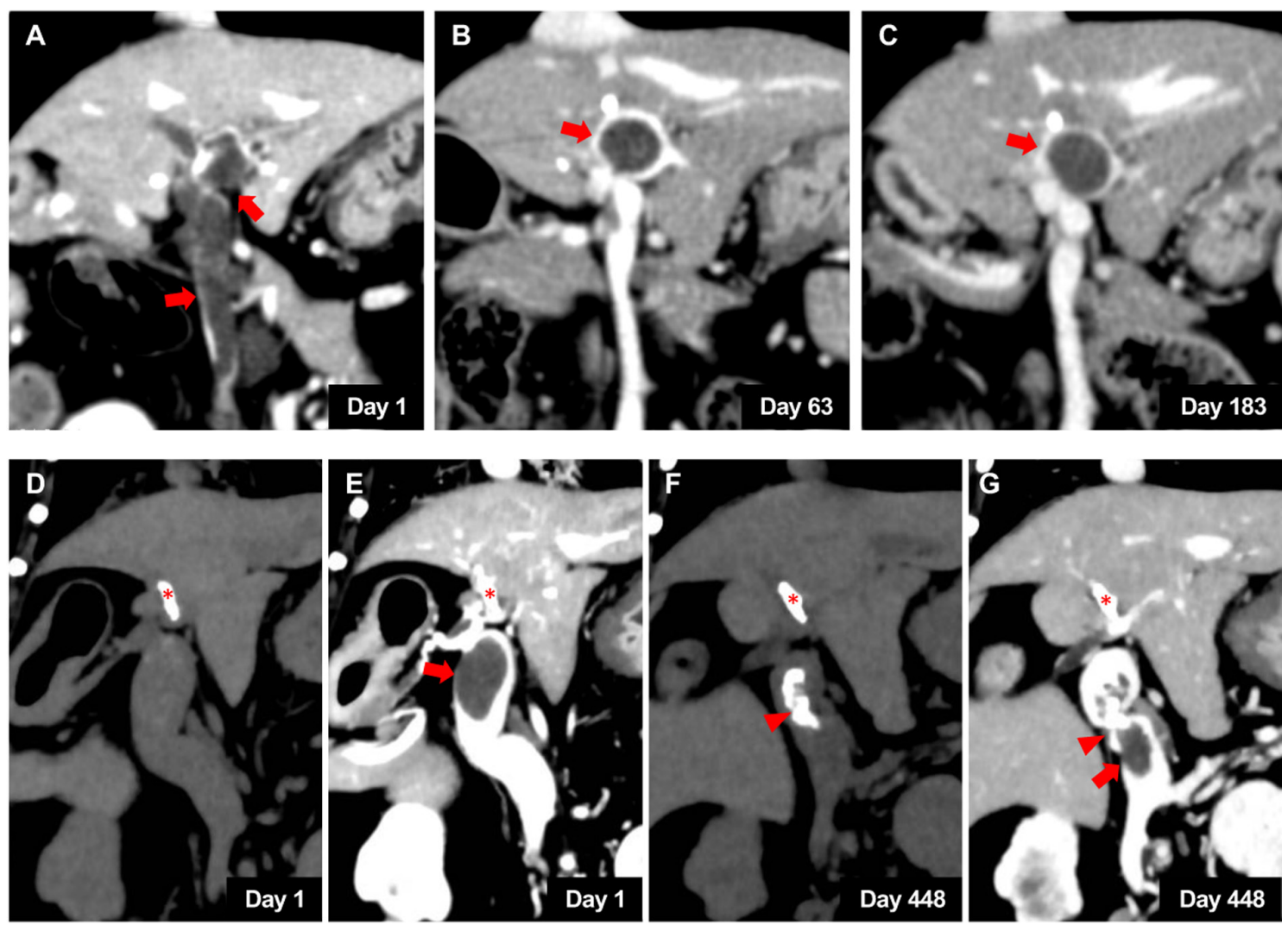

Fig. 3. Dorsal CT images of follow-up CT angiography. CT images of Dog 13 obtained on day 1 (A), day 63 (B), and day 183 (C). The thrombus (arrow) size was markedly decreased in size on day 63, and a mild increase can be detected on day 183. CT images of Dog 2 obtained on day 1 (D, E) and day 448 (F, G). The organized area of the thrombus was increased on day 448 (arrowhead). Arrow, portal vein thrombosis; Asterisk, organized thrombus; D and F, plane images; $\mathrm{E}$ and $\mathrm{G}$, contrast enhanced images.

is a good predictor of portal hypertension [2], and the asymmetry of the liver lobes may be a result of decreased blood flow due to portal obstruction. Thus, CT can provide evidence of remote thrombus formation that prompt clinicians to perform antithrombotic therapy and yield information to understand the pathophysiological changes caused by the thrombus.

In the present study, D-dimer concentration was elevated in all dogs measured. In addition, dogs with thrombosis in multiple locations presented with statistically higher D-dimer concentration compared to single PVT dogs in the present study. D-dimer is a stable end product of fibrin degradation, and fibrin is converted from fibrinogen by activated thrombin; thus, the elevation of D-dimer concentration reflects fibrinolysis [13]. In humans, serum D-dimer concentration has been shown to be significantly high in patients with PVT in conditions such as liver cirrhosis and hepatocellular carcinoma [13, 25]. In veterinary medicine, elevated plasma D-dimer concentration has been reported in thromboembolic diseases [14, 15, 21]; however, recent studies on dogs with PVT provide limited data on the relationship between PVT and D-dimer concentration [7, 18]. D-dimer concentration was significantly higher in dogs with thromboembolic disease and critically ill dogs with postmortem evidence of thrombosis [15, 21]. Additionally, there was a positive correlation between D-dimer concentration and the number of thrombi [21]. Thus, the elevation of D-dimer concentrations may support the suspicion of thrombosis of the portal vein in dogs.

In the present study, follow-up data were obtained for all dogs, including 10 dogs undergoing treatment. Further, in two dogs, we reexamined the size and organization of the thrombus using CT angiography. In these dogs, the morphological changes in the thrombus were detected only by CT angiography. In humans, CT angiography is commonly used to confirm the change in thrombus size or location during follow-up, and the result is used for treatment evaluation $[3,4,8]$. Since abdominal ultrasound was insufficient in detecting the size and number of the thrombus compared with CT angiography, we believe that CT examination is a more reliable tool for follow-up and possibly for treatment evaluation in dogs with PVT.

There are some limitations to the present study. The present study was a retrospective study including a small case number of dogs. Only dogs suspected of gastroenterological disorders were included, and all dogs were classified as chronic PVT. This may have led to a bias in the results. Furthermore, follow-up assessments using CT angiography were performed in only two dogs and the treatment was not standardized.

In conclusion, $\mathrm{CT}$ angiography readily visualized the specific location and number of PVT. CT angiography can be applied to facilitate the definitive diagnosis and improve our understanding of the characteristics of PVT in dogs. Therefore, dogs suspected of PVT should undergo CT angiography even when thrombosis in the portal vein is not diagnosed under ultrasound, especially when 
the D-dimer concentration is increased. In addition, periodical CT examination may be useful for the follow-up evaluation of PVT in dogs.

\section{REFERENCES}

1. Bertolini, G. 2010. Acquired portal collateral circulation in the dog and cat. Vet. Radiol. Ultrasound 51: 25-33. [Medline] [CrossRef]

2. Buob, S., Johnston, A. N. and Webster, C. R. 2011. Portal hypertension: pathophysiology, diagnosis, and treatment. J. Vet. Intern. Med. 25: 169-186. [Medline] [CrossRef]

3. Cai, M., Zhu, K., Huang, W., Meng, X., He, K., Zhou, B., Guo, Y., Chen, J. and Shan, H. 2013. Portal vein thrombosis after partial splenic embolization in liver cirrhosis: efficacy of anticoagulation and long-term follow-up. J. Vasc. Interv. Radiol. 24: 1808-1816. [Medline] [CrossRef]

4. Chung, J. W., Kim, G. H., Lee, J. H., Ok, K. S., Jang, E. S., Jeong, S. H. and Kim, J. W. 2014. Safety, efficacy, and response predictors of anticoagulation for the treatment of nonmalignant portal-vein thrombosis in patients with cirrhosis: a propensity score matching analysis. Clin. Mol. Hepatol. 20: 384-391. [Medline] [CrossRef]

5. DeLeve, L. D., Valla, D. C., Garcia-Tsao G., American Association for the Study Liver Diseases 2009. Vascular disorders of the liver. Hepatology 49: 1729-1764. [Medline] [CrossRef]

6. Díaz Espiñeira, M. M., Vink-Nooteboom, M., Van den Ingh, T. S. and Rothuizen, J. 1999. Thrombosis of the portal vein in a miniature schnauzer. J. Small Anim. Pract. 40: 540-543. [Medline] [CrossRef]

7. French, J. M., Twedt, D. C., Rao, S. and Marolf, A. J. 2019. Computed tomographic angiography and ultrasonography in the diagnosis and evaluation of acute pancreatitis in dogs. J. Vet. Intern. Med. 33: 79-88. [Medline]

8. Fujiyama, S., Saitoh, S., Kawamura, Y., Sezaki, H., Hosaka, T., Akuta, N., Kobayashi, M., Suzuki, Y., Suzuki, F., Arase, Y., Ikeda, K. and Kumada, H. 2017. Portal vein thrombosis in liver cirrhosis: incidence, management, and outcome. BMC Gastroenterol. 17: 112. [Medline] [CrossRef]

9. Goggs, R., Benigni, L., Fuentes, V. L. and Chan, D. L. 2009. Pulmonary thromboembolism. J. Vet. Emerg. Crit. Care (San Antonio) 19: 30-52. [Medline] [CrossRef]

10. Kutara, K., Seki, M., Ishikawa, C., Sakai, M., Kagawa, Y., Iida, G., Ishigaki, K., Teshima, K., Edamura, K., Nakayama, T. and Asano, K. 2014. Triple-phase helical computed tomography in dogs with hepatic masses. Vet. Radiol. Ultrasound 55: 7-15. [Medline] [CrossRef]

11. Laurenson, M. P., Hopper, K., Herrera, M. A. and Johnson, E. G. 2010. Concurrent diseases and conditions in dogs with splenic vein thrombosis. J. Vet. Intern. Med. 24: 1298-1304. [Medline] [CrossRef]

12. Machida, T., Kokubu, H., Matsuda, K., Miyoshi, K. and Uchida, E. 2010. Clinical use of D-dimer measurement for the diagnosis of disseminated intravascular coagulation in dogs. J. Vet. Med. Sci. 72: 1301-1306. [Medline] [CrossRef]

13. Malaguarnera, M., Latteri, S., Bertino, G., Madeddu, R., Catania, V. E., Currò, G., Borzì, A. M., Drago, F. and Malaguarnera, G. 2018. D-dimer plasmatic levels as a marker for diagnosis and prognosis of hepatocellular carcinoma patients with portal vein thrombosis. Clin. Exp. Gastroenterol. 11: 373-380. [Medline] [CrossRef]

14. Marschner, C. B., Kristensen, A. T., Rozanski, E. A., McEvoy, F. J., Kühnel, L., Taeymans, O., de Laforcade, A., Sato, A. F. and Wiinberg, B. 2017. Diagnosis of canine pulmonary thromboembolism by computed tomography and mathematical modelling using haemostatic and inflammatory variables. Vet. J. 229: 6-12. [Medline] [CrossRef]

15. Nelson, O. L. and Andreasen, C. 2003. The utility of plasma D-dimer to identify thromboembolic disease in dogs. J. Vet. Intern. Med. 17: 830-834. [Medline] [CrossRef]

16. Or, M., Ishigaki, K., de Rooster, H., Kutara, K. and Asano, K. 2016. Determination of porto-azygos shunt anatomy in dogs by computed tomography angiography. Vet. Surg. 45: 1005-1012. [Medline] [CrossRef]

17. Ponziani, F. R., Zocco, M. A., Campanale, C., Rinninella, E., Tortora, A., Di Maurizio, L., Bombardieri, G., De Cristofaro, R., De Gaetano, A. M., Landolfi, R. and Gasbarrini, A. 2010. Portal vein thrombosis: insight into physiopathology, diagnosis, and treatment. World J. Gastroenterol. 16: 143-155. [Medline] [CrossRef]

18. Respess, M., O’Toole, T. E., Taeymans, O., Rogers, C. L., Johnston, A. and Webster, C. R. 2012. Portal vein thrombosis in 33 dogs: 1998-2011. J. Vet. Intern. Med. 26: 230-237. [Medline] [CrossRef]

19. Sakamoto, Y., Sakai, M. and Watari, T. 2017. Portal vein/aorta ratio in dogs with acquired portosystemic collaterals. J. Vet. Intern. Med. 31: 1382-1387. [Medline] [CrossRef]

20. Specchi, S., Pey, P., Ledda, G., Lustgarten, M., Thrall, D. and Bertolini, G. 2015. Computed tomographic and ultrasonographic characteristics of cavernous transformation of the obstructed portal vein in small animals. Vet. Radiol. Ultrasound 56: 511-519. [Medline] [CrossRef]

21. Thawley, V. J., Sánchez, M. D., Drobatz, K. J. and King, L. G. 2016. Retrospective comparison of thromboelastography results to postmortem evidence of thrombosis in critically ill dogs: 39 cases (2005-2010). J. Vet. Emerg. Crit. Care (San Antonio) 26: 428-436. [Medline] [CrossRef]

22. Van Winkle, T. J. and Bruce, E. 1993. Thrombosis of the portal vein in eleven dogs. Vet. Pathol. 30: 28-35. [Medline] [CrossRef]

23. von Köckritz, L., De Gottardi, A., Trebicka, J. and Praktiknjo, M. 2017. Portal vein thrombosis in patients with cirrhosis. Gastroenterol. Rep. (Oxf.) 5: 148-156. [Medline] [CrossRef]

24. Wang, L., Li, Z. S., Lu, J. P., Wang, F., Liu, Q. and Tian, J. M. 2008. Cavernous transformation of the portal vein: three-dimensional dynamic contrast-enhanced MR angiography. Abdom. Imaging 33: 463-468. [Medline] [CrossRef]

25. Zhang, D. L., Hao, J. Y. and Yang, N. 2013. Value of D-dimer and protein S for diagnosis of portal vein thrombosis in patients with liver cirrhosis. J. Int. Med. Res. 41: 664-672. [Medline] [CrossRef]

26. Zhang, M. M., Pu, C. L., Li, Y. C. and Guo, C. B. 2011. Sixty-four-slice computed tomography in surgical strategy of portal vein cavernous transformation. World J. Gastroenterol. 17: 4334-4338. [Medline] [CrossRef] 